\title{
SURFACE MOUNTED HEAT FLUX SENSORS
}

\author{
G. J. WEIR
}

(Received 12 December 1984; revised 12 April 1985)

\begin{abstract}
The dual integral equations describing heat flow about a circular Heat Flux Sensor on the surface of a layered medium are derived and discussed, together with the extent to which the Heat Flux Sensor measures the heat flow which would occur in the absence of a Heat Flux Sensor. An asymptotic analysis provides new analytical results supporting those derived previously by numerical methods.

It is suggested that some properties of the general problem of a Heat Flux Sensor on the surface of a multiply-layered medium can be approximated by a lumped-parameter model depending on only four non-dimensional numbers; namely, two non-dimensional linear heat transfer coefficients, and essentially two non-dimensional thermal resistances. Some support for the lumped parameter model is provided
\end{abstract}

\section{Introduction}

What are the main factors determining the relative error of a Heat Flux Sensor (HFS) on a layered floor? How does the relative error of a HFS depend quantatively on these factors? These two questions are of interest to the measurement of heat flow through buildings, since a HFS typically measures the heat flow through itself, whereas one usually requires the heat flow which would occur in the absence of a HFS. Furthermore, the response of a HFS depends on the geometry and composition of the wall, and so an analytic approach may be useful in summarising the results of numerical models of a HFS.

The aim of this paper is to develop an analytic model of an idealised HFS in order to address the questions above. We assume for simplicity that the HFS is circular, and infinitesimally thin. Consequently the mathematical problem to be

\footnotetext{
'Applied Mathematics Division, Department of Scientific and Industral Research. P.P. Box 1335. Wellington, New Zealand.

S Copyright Australian Mathematical Society 1986, Serial-fee code 0334-2700/86
} 
treated resembles one discussed previously by the author (Weir [6]), but extends our earlier results in three ways. Firstly, a layered medium is introduced, and the resulting mathematical structures detailed. Secondly, the leading term in an asymptotic expansion of an important integral is derived, and thirdly, four non-dimensional numbers are obtained which should be important in quantifying the relative error of an HFS.

It is important to distinguish between surface-mounted and embedded heat flux sensors, which are obviously positioned respectively on and within the conducting medium. Some factors which may be important for the performance of a surface-mounted HFS are the finite thickness of the conducting medium (floor, wall, etc.), significant transverse temperature gradients induced by the presence of the HFS, significant changes longitudinally in the thermal conductivity of the medium and surface heat transfer coefficients. Some of the factors above are omitted in discussions of embedded HFSs [4], and for the remainder of this paper we shall understand our HFS to be surface-mounted.

Perhaps the main physical difference between surface-mounted and embedded HFSs is that for mismatched surface heat transfer coefficients the influence of a surface-mounted HFS is non-zero, independent of its thickness, whereas the influence of an infinitesimally thin embedded HFS is zero. Mathematically, the radiation boundary conditions introduce new length scales which are missing for an embedded HFS.

\section{Model formulation}

A thin circular Heat Flux Sensor (HFS) of radius $R_{0}$ lies on the surface of a horizontally layered floor of unbounded horizontal extent. A steady (though spatially variable) heat flow occurs from the lower surface $Z=-Z_{N}$ to $Z=0$, and we shall discuss the extent to which the HFS records the heat flow which would occur in the absence of a HFS.

In a cylindrical coordinate system $(R, Z)$ (see Figure 1), the model equations are

$$
\begin{aligned}
\nabla^{2} T= & 0, \quad Z<0, \\
T= & T_{N}, \quad Z=-Z_{N}, \\
K_{1} T,_{Z}= & -h_{0}\left(T-T_{0}\right), \quad Z=0, \quad 0<R<R_{0}, \\
K_{1} T,_{Z}= & -h_{1}\left(T-T_{0}\right), \quad Z=0, \quad R>R_{0}, \\
& T \text { and } K T,_{Z} \text { continuous, }
\end{aligned}
$$


where $T$ is temperature, $K_{t}$ thermal conductivity of layer $i(i=1, \ldots, N), h_{0}$ and $h_{1}$ the linear heat transfer coefficients of the HFS and floor surface respectively, and $T_{0}$ and $T_{N}$ are constants. The thermal conductivity $K_{t}$ is assumed constant within the $i$ th layer which occupies $-Z_{t}<Z<-Z_{t-1}$, and $Z_{0}$ equals zero.

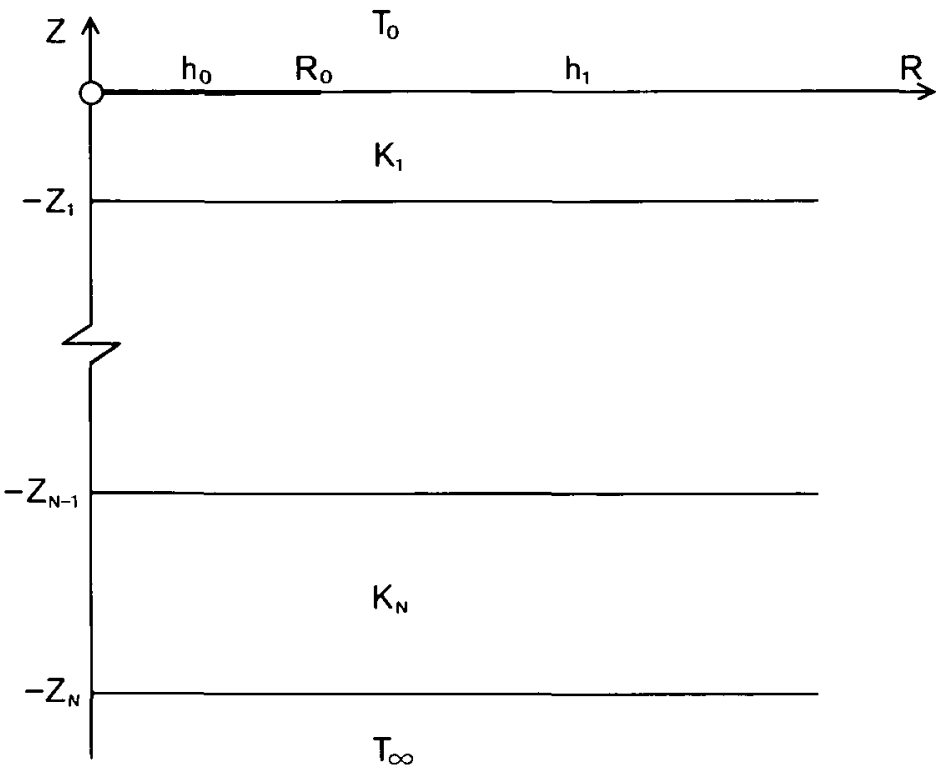

Figure 1. Schematic diagram of a circular Heat Flux Sensor on an $\mathrm{N}$-layered medium.

The definitions

$$
\begin{aligned}
Q_{\infty} & =\left(T_{N}-T_{0}\right) /\left[\frac{1}{h_{1}}+\sum_{i=1}^{N} \frac{\left(Z_{i}-Z_{t-1}\right)}{K_{i}}\right], \\
T & =T_{u}+\frac{Q_{\infty} R_{0}}{K_{1}}\left(1-\frac{h_{0}}{h_{1}}\right) \psi, \\
T_{u} & =T_{0}-Q_{\infty} / h_{1}-Q_{\infty} Z / K_{1}, \quad-Z_{1}<Z<0, \\
\left(R, Z, Z_{t}\right) & =R_{0}\left(r, z, z_{i}\right), \\
\alpha & =h_{0} R_{0} / K_{1}, \\
\beta & =h_{1} R_{0} / K_{1},
\end{aligned}
$$


yield the following non-dimensional equations

$$
\begin{gathered}
\nabla^{2} \psi=0, \quad-z_{N}<z<0, \\
\psi=0, \quad z=-z_{N}, \\
\psi \text { and } K \psi_{, z} \text { continuous, } \\
\psi, z=-\alpha \psi+1, \quad z=0, \quad 0<r<1, \\
\psi_{, z}=-\beta \psi, \quad z=0, \quad r>1,
\end{gathered}
$$

where $T_{u}$ is the piecewise linear temperature distribution which results in the absence of a HFS.

It is convenient to use Bessel Transforms

$$
\mathscr{J}_{0} \psi=\int_{0}^{\infty} J_{0}(r p) \psi r d r
$$

to define

$$
G(p)=\mathscr{J}_{0} \psi / \mathscr{J}_{0} \psi, \text { on } z=0
$$

and

$$
F(p)=p \mathscr{J}_{0}\left(\psi,_{z}+\beta \psi\right) \quad \text { on } z=0
$$

Hence

$$
J_{0} \psi=F G /(p+\beta p G) \text { on } z=0
$$

and

$$
\mathscr{J}_{0} \psi_{z}=F /(p+\beta p G) \quad \text { on } z=0 .
$$

Application of Bessel Transforms to the boundary conditions in (6) then yield the dual integral equations

$$
\begin{array}{rlrl}
\int_{0}^{\infty} F(p) J_{0}(r p) d p & =0, & r>1, \\
\int_{0}^{\infty} F(p)\left(\frac{1+\alpha G}{1+\beta G}\right) J_{0}(r p) d p=1, & 0<r<1 .
\end{array}
$$

When $\alpha=\beta$ the solution of (11) and (12) is $F(p)=J_{1}(p)$, and Tranter's method [2] suggests setting

$$
F(p)=\sum_{m=0}^{\infty} a_{m} J_{2 m+1}(p),
$$

which automatically satisfies (11), and then finite Legendre transforms

$$
\begin{gathered}
\int_{0}^{1} 2 r P_{n}\left(1-2 r^{2}\right) d r=\delta_{n, 0} \\
\int_{0}^{1} r J_{0}(r p) P_{n}\left(1-2 r^{2}\right) d r=p^{-1} J_{2 n+1}(p)
\end{gathered}
$$

and the Weber-Schafheitlin integral

$$
\int_{0}^{\infty} J_{2 m+1}(p) J_{2 n+1}(p) d p / p=\delta_{n, m} /(2+4 n)
$$


reduces (12) to the solution of the algebraic system

$$
\alpha a_{n}+(\beta-\alpha)(2+4 n) \sum_{m=0}^{\infty} a_{m} K_{m n}=\beta \delta_{n, 0},
$$

where

$$
K_{m n}=\int_{0}^{\infty} \frac{J_{2 m+1}(p) J_{2 n+1}(p) d p}{(p+\beta p G)} .
$$

The function $K_{m n}$ is independent of $\alpha$. Intuitively, the series (13) appears especially appropriate for $(\alpha / \beta)$ approximately equalling unity.

A simple expression for the surface values of $\psi$ on $0<r<1$ can be obtained from (9), since on $z=0$

$$
\psi=\int_{0}^{\infty} \frac{F J_{0}(r p) d p}{\beta}-\int_{0}^{\infty} \frac{F J_{0}(r p) p d p}{\beta(p+\beta p G)},
$$

and so from (13)-(15), on $z=0$,

$$
(\beta-\alpha)(2+4 n) \int_{0}^{1} \psi r P_{n}\left(1-2 r^{2}\right) d r=a_{n}-\delta_{n, 0} .
$$

Consequently the orthogonality of the Legendre functions yields

$$
1+(\beta-\alpha) \psi=\sum_{0}^{\infty} a_{m} P_{m}\left(1-2 r^{2}\right) \quad \text { on } z=0,0<r<1 .
$$

In particular, $\bar{\psi}$, the mean value of $\psi$ over the HFS, is related linearly to $a_{0}$,

$$
a_{0}=1+(\beta-\alpha) \bar{\psi}
$$

Finally, we define the relative error, RE, of a HFS as the difference between $Q_{\infty}$ and the mean heat flux through the HFS, relative to $Q_{\infty}$, or

$$
\begin{aligned}
\mathrm{RE} & =1+\frac{1}{\pi Q_{\infty} R_{0}^{2}} \int_{0}^{R_{0}} 2 \pi R K_{1} T,{ }_{z} d R \quad \text { on } Z=0, \\
& =2\left(1-\frac{\alpha}{\beta}\right) \int_{0}^{1} \psi,{ }_{z} r d r \text { on } z=0, \\
& =2\left(1-\frac{\alpha}{\beta}\right) \int_{0}^{1} r d r \int_{0}^{\infty} \frac{F(p) J_{0}(r p) d p}{(1+\beta G)} \\
& =2\left(1-\frac{\alpha}{\beta}\right) \sum_{m=0}^{\infty} a_{m} K_{m 0} \\
& =1-\frac{\alpha a_{0}}{\beta} .
\end{aligned}
$$

\section{The function $G(p)$}

To determine the functions $K_{m n}$ defined in (15) we must first calculate the function $G(p)$ defined in (7). Firstly, since $\psi$ satisfies Laplace's Equation, within 
each layer there are functions $\left(V_{i}, W_{i}\right)$ independent of $z$ satisfying

$$
\mathscr{J}_{0} \psi=V_{1} e^{p z}+W_{1} e^{-p z}, \quad-z_{1}<z<-z_{i-1},
$$

where $z_{0}=0$, and $i=1, \ldots, N$.

Application of the boundary conditions yield

$$
\begin{aligned}
V_{N} e^{-p z_{N}}+W_{N} e^{p z_{N}} & =0 \\
V_{1} e^{-p z_{1}}+W_{1} e^{p z_{1}} & =V_{i+1} e^{-p z_{t}}+W_{t+1} e^{p z_{1}}, \\
K_{l}\left(V_{l} e^{-p z_{t}}-W_{1} e^{p z_{1}}\right) & =K_{t+1}\left(V_{1+1} e^{-p z_{1}}-W_{1+1} e^{p z_{1}}\right),
\end{aligned}
$$

where $i=1, \ldots, N-1$, and from (7) and (18) we require

$$
G=\frac{V_{1}+W_{1}}{p\left(V_{1}-W_{1}\right)}
$$

By defining

$$
\left(\begin{array}{l}
v_{1} \\
w_{1}
\end{array}\right)=\frac{1}{2}\left(e^{-p z_{1} V_{1}} \mp e^{p z_{1} W_{1}}\right)
$$

(19) becomes

$$
\begin{aligned}
& w_{N}=0 \text {, } \\
& \left(\begin{array}{l}
v_{\imath} \\
w_{l}
\end{array}\right)=\left(\begin{array}{cc}
\left(K_{i+1} / K_{l}\right) \cosh p\left(z_{i+1}-z_{\imath}\right) & \left(K_{i+1} / K_{\imath}\right) \sinh p\left(z_{i+1}-z_{i}\right) \\
\sinh p\left(z_{i+1}-z_{\imath}\right) & \cosh p\left(z_{\imath+1}-z_{\imath}\right)
\end{array}\right)\left(\begin{array}{l}
v_{1+1} \\
w_{i+1}
\end{array}\right) \\
& \equiv L_{t}\left(\begin{array}{l}
v_{i+1} \\
w_{t+1}
\end{array}\right) \\
& G=\frac{v_{1} \sinh p z_{1}+w_{1} \cosh p z_{1}}{p v_{1} \cosh p z_{1}+p w_{1} \sinh p z_{1}},
\end{aligned}
$$

where the $2 \times 2$ matrix $L_{1}$ is defined between (20b) and (20c). Hence

$$
G=w_{0} / v_{0} \text {, }
$$

where

$$
\begin{aligned}
\left(\begin{array}{l}
v_{0} \\
w_{0}
\end{array}\right) & =v_{N} \prod_{t=0}^{N-1} L_{\iota}\left(\begin{array}{l}
1 \\
0
\end{array}\right), \\
K_{0} & =K_{1} / p,
\end{aligned}
$$

and the indices $i$ in the product of $L_{1}$ matrices are arranged in increasing order to the right. The definition of $G$ in (21)-(23) shows (by induction) that under the transformation $z_{j} \rightarrow-z_{j}, v_{i}$ are even and $w_{\iota}$ odd, for $i=0, \ldots, N-1$; and so $G$ is odd under $z_{j} \rightarrow-z_{j}$. Similarly, $G$ is even under the transformation $p \rightarrow-p$.

The product of the $L_{\imath}$ matrices involves addition and multiplication of nonnegative quantities, and so $G$ is non-negative. For $p$ sufficiently large, $\tanh \left(p z_{1}\right)$ tends to unity, and so from (20d)

$$
\lim _{p \rightarrow \infty} p G(p)=1
$$


Similarly,

$$
\lim _{z_{1} \rightarrow \infty} p G=1, \quad p \neq 0 .
$$

As $p$ tends to zero, the product of $L_{l}$ yields

$$
\begin{aligned}
& v_{1}=K_{N} v_{N} / K_{1}+O(p), \\
& w_{1}=p K_{N} v_{N}\left[\sum_{\imath=2}^{N}\left(\frac{z_{\imath}-z_{\imath-1}}{K_{\imath}}\right)+O\left(p^{2}\right)\right]
\end{aligned}
$$

and so from $(20 \mathrm{~d})$

$$
\lim _{p \rightarrow 0} G(p)=K_{1}\left[\sum_{t=1}^{N} \frac{\left(z_{t}-z_{t-1}\right)}{K_{t}}\right] .
$$

As an alternative to the algebraic derivation of $G$ above, one can proceed geometrically, since the matrices $L_{1}$ can be interpreted as geometric transformations of the points $\left(v_{1+1}, w_{1+1}\right)$ into $\left(v_{l}, w_{l}\right)$ in a two-dimensional space $(v, w)$. From (20b), $L_{t}$ is the product of a Lorentz transformation (preserving $v^{2}-w^{2}$ ), followed by a shear in the $v$ direction. The Lorentz transformation "rotates" points along a rectangular hyperbola in $(v, w)$ space, whereas the shear can allow points to cross the line $v=w$.

We shall assume initially that

$$
K_{0}<K_{1}<\cdots<K_{N}
$$

Then the definition

$$
\tanh \theta_{t}=w_{1} / v_{i}
$$

together with (20), gives

$$
\begin{aligned}
\theta_{N} & =0, \\
\tanh \theta_{i} & =\frac{K_{l}}{K_{i+1}} \tanh \left[\theta_{i+1}+p\left(z_{i+1}-z_{i}\right)\right], \quad i=0, \ldots, N-1, \\
G & =\tanh \theta_{0} .
\end{aligned}
$$

Expanding the right-hand side of (27) recursively so as to eliminate all of the $\theta_{t+1}$ yields an equation for $G$ involving only expressions of the form $K_{i}, K_{t+1}$ and $\tanh p\left(z_{i+1}-z_{i}\right)$ for $i=0, \ldots, N-1$, and this equation is obviously valid if the initial restriction on the magnitudes of $K_{1}$ is now dropped. This is perhaps the simplest method for calculating $G$.

There is an analogy with the above results and some from electromagnetism. In Electromagnetic Theory [5] the ratio $E_{x} / H_{y}$ on the surface $z=0$ is called the surface impedance, and since $E_{x}$ is often proportional to the vertical gradient of $H_{y}$ we see from (7) that $G$ is analogous to the surface impedance, and $\left(v_{i}, w_{i}\right)$ are analogous to transmitted and reflected waves. 
Similarly, special "angular" relationships are possible, analogous to those in Optics, since the line $v=w$ divides one set of rectangular hyperbole (with $w<v$ ) from another (with $v<w$ ). The line $v=w$ is analogous to the light cone in Special Relativity Theory. We seek the conditions under which a non-unity ratio $w_{i+1} / v_{t+1}$ is mapped under $L_{1}$ into unity.

If $w_{t+1} / v_{i+1}$ and $K_{t+1} / K_{i}$ are both greater than unity, we define

$$
\begin{aligned}
w_{t+1} / v_{t+1} & =\operatorname{coth} \eta_{t+1}, \\
K_{t+1} / K_{\imath} & =\operatorname{coth} \phi_{t+1} .
\end{aligned}
$$

Then $w_{t} / v_{1}$ is unity, provided

$$
\phi_{t+1}=\eta_{t+1}+p\left(z_{t+1}-z_{t}\right) .
$$

The same condition follows if

$$
\tanh \eta_{t+1}=w_{t+1} / v_{t+1}<1,
$$

and

$$
\tanh \phi_{t+1}=K_{t+1} / K_{t}<1 .
$$

Consequently, if $\eta_{1+1} \leqslant \phi_{1+1}$, there is always one value of $p$ satisfying (28). Furthermore, from (20d), if for example $v_{1}=w_{1}$, then $G$ has the same value there as when $z_{1}$ is infinite.

\section{A uniform half-plane $\left(z_{1}=\infty\right)$}

When $z_{1}$ is infinite the problem above reduces to that of a HFS on a uniform half-plane, and from (25) and (15) $K_{m n}$ reduces to $J_{m n}$, where

$$
J_{m n}(\beta)=\int_{0}^{\infty} \frac{J_{2 m+1}(p) J_{2 n+1}(p) d p}{p+\beta} .
$$

The behaviour of (29) is known [6] for small $\beta$. In particular,

$$
J_{00}(\beta)=\frac{1}{2}-4 \beta / 3 \pi+O\left(\beta^{2} \ln \beta\right),
$$

and provided $a_{0}$ is the dominant term,

$$
\begin{aligned}
a_{n} & \cong \delta_{n, 0}+(-1)^{n+1}(\beta-\alpha) /\left[\pi\left(n+\frac{3}{2}\right)\left(n^{2}-\frac{1}{4}\right)\right], \\
\mathrm{RE} & \cong\left(1-\frac{\alpha}{\beta}\right)\left(1-\frac{8 \alpha}{3 \pi}\right), \\
\beta & \ll 3 \pi / 8 \cong 1.18 .
\end{aligned}
$$

Consequently the problem of small $\beta$ (and $\alpha$ ) has been solved, and it remains to discuss perhaps the more important problem of large $\beta$. 
The leading term in an asymptotic expansion for $J_{m n}(\beta)$ can be obtained (for moderate $m$ and $n$ ) by considering the following expression for $J_{m n}$

$$
\begin{aligned}
J_{m n}(\beta)= & \frac{\pi}{2 i} \int_{c-1 \infty}^{c+\infty} \frac{\Gamma(2 m+2 n+2 s+3)\left(\frac{\beta}{2}\right)^{2 m+2 n+2 s+2} d s}{M(s) \sin 2 \pi s \sin \pi s} \\
& +\frac{1}{2 \pi} \oint \frac{N(y) d y}{D(y)},
\end{aligned}
$$

where $-m-n-2<c<-m-n-3 / 2$,

$$
\begin{aligned}
& M(s)=\Gamma(s+1) \Gamma(2 m+s+2) \Gamma(2 n+s+2) \Gamma(2 m+2 n+s+3), \\
& N(y)=\frac{(-1)^{m+n+1} \pi^{2}(\beta / 2)^{2 y-1}}{\Gamma\left(y-m-n-\frac{1}{2}\right) \Gamma\left(m-n+y+\frac{1}{2}\right) \Gamma\left(n-m+\frac{1}{2}+y\right)}, \\
& D(y)=\cos \pi y \sin 2 \pi y \Gamma\left(m+n+\frac{3}{2}+y\right) / \Gamma(2 y),
\end{aligned}
$$

which follows by displacing a published line integral (Equation 21, [6]) one half integer to the left. The closed line integral in (30) is a small anti-clockwise circle surrounding the origin, where $D(y)$ contributes a double pole, and $N(y)$ is analytic. We find

$$
\begin{aligned}
\frac{1}{2 \pi} \oint \frac{N(y) d y}{D(y)}=\frac{(-1)^{m+n}}{\pi \beta}[\ln 8 \beta+\gamma & -2 \sum_{i=0}^{m+n}\left(\frac{1}{2 i+1}\right) \\
& \left.-2\left(1-\delta_{m, n}\right)\left(\sum_{i=0}^{|m-n|-1} \frac{1}{2 i+1}\right)\right],
\end{aligned}
$$

where $\gamma$ is Euler's constant. Equation (31) is the leading term in an asymptotic expansion for $J_{m n}(\beta)$, since the remaining line integral in (30) is $O\left(\beta^{-\lambda}\right)$, where $-2<\lambda<-1$, which follows from a well-known property (6.1.45 in [1]) of the Gamma function.

Consequently, for large $\beta$,

$$
J_{00}(\beta) \cong \frac{1}{\pi \beta}(\ln \beta+0.657),
$$

and provided $a_{0}$ is the dominant term,

$$
\begin{aligned}
a_{0} & \cong \beta /\left[\alpha+2(\beta-\alpha) J_{00}(\beta)\right] \\
\mathrm{RE} & \cong 2(\beta-\alpha) J_{00}(\beta) /\left[\alpha+2(\beta-\alpha) J_{00}(\beta)\right]
\end{aligned}
$$

The Bessel transform expression for $J_{00}(\beta)$ shows that for large $\beta, J_{00}(\beta)$ is a monotonically decreasing function of $\beta$. However, the approximation above for $J_{00}(\beta)$ has a maximum at approximately 1.41 , and so we require

$$
\beta \gg 1.41
$$

for $\beta$ to be considered large. 
Higher order contributions to the asymptotic expansion for $J_{m n}(\beta)$ are obtained by moving the first line integral in (30) to the left in successive half integer steps, but for large $\beta$, the leading term given in (31) should be sufficient.

\section{Lumped parameter model}

For a general $N$-layer model the integrals to be performed are complicated, which suggests the introduction of an approximation. Our main aim in using approximate expressions is to derive dimensionless numbers useful in the classification of numerical results.

For a layered floor of finite thickness the Bessel transforms in (15) are perhaps best treated numerically, but for infinitely thick homogeneous floors, the integrals $J_{m n}(\beta)$ in (29) have been determined analytically. Consequently our approach is to replace some difficult Bessel transform for a layered floor by another simpler transform of the type occuring for homogeneous unbounded floors.

The primary motivation for introducing such approximations is to provide a theoretical justification for recent empirical results by $H$. Trethowen and $N$. Isaacs [3] which indicate that the relative error for many practical Heat Flux Sensors on the surface of a doubly layered medium can be approximately described by one non-dimensional number, whereas an exact analysis suggests five non-dimensional numbers as being important. We shall proceed by deriving an approximate expression for the relative error of a HFS, which has yielded similar results to the previous fully empirical approach. It is hoped to report these findings at a later date.

The first approximation we consider is

$$
\frac{1+\alpha G}{1+\beta G} \cong \frac{p+\alpha^{*}}{p+\beta^{*}}
$$

where

$$
\begin{aligned}
\alpha^{*} & =\frac{p^{*} G\left(p^{*}\right)[1+\alpha G(0)]}{\left[G(0)-G\left(p^{*}\right)\right]}, \\
\beta^{*} & =\frac{p^{*} G\left(p^{*}\right)[1+\beta G(0)]}{\left[G(0)-G\left(p^{*}\right)\right]} .
\end{aligned}
$$

By construction, equality holds in (32) when $p$ equals $0, p^{*}$ or $\infty$, and we are assuming that none of the layers are infinitely thick. When one of the layers is infinitely thick, the form of approximation in (32) still holds (from the behaviour for large $p$ ), but now $\alpha^{*}$ and $\beta^{*}$ are obtained by considering the limiting behaviour of the left-hand side of (32) for two values of $p$ (perhaps 0 and 1). Only finitely thick floors will be considered in the following discussion. 
We shall now show inductively that $G$ is a monotonically decreasing function of $p$. Firstly, for a finitely thick uniform layer, $G$ is simply $p^{-1} \tanh p z_{1}$, and so for $N=1, G$ is a monotonically decreasing function of $p$. The inductive step is established by considering an $N$-layered floor to be a composite of the lowermost $N-1$ layers plus the upper layer. Then, if $G_{N-1}$ is the corresponding $G$ function for the lower $N-1$ layers, from (20)

$$
G_{N-1}=\frac{v_{2} \sinh p\left(z_{2}-z_{1}\right)+w_{2} \cosh p\left(z_{2}-z_{1}\right)}{p\left[v_{2} \cosh p\left(z_{2}-z_{1}\right)+w_{2} \sinh p\left(z_{2}-z_{1}\right)\right]}
$$

and

$$
G=\frac{K_{2} \sinh p z_{1}+p K_{1} \cosh p z_{1} G_{N-1}}{p\left(K_{2} \cosh p z_{1}+p K_{1} \sinh p z_{1} G_{N-1}\right)}
$$

Differentiating (35) with respect to $p$, observing that $(\sinh x \cosh x-x)$ is positive for positive $x$, and assuming that $G_{N-1}$ is monotonically decreasing then shows that $G$ is indeed monotonically decreasing in $p$.

Consequently, from (33) and (34), both $\left(\alpha^{*}-\beta^{*}\right)$ and $(\alpha-\beta)$ have the same sign, and so the obvious monotonicity on the right-hand side of (32) also occurs on the left-hand side of (32). Hence the right-hand side of (32) does indeed provide a rough approximation to the left-hand side, and clearly the use of more general Padé approximates on the right will improve the approximation. However, in what follows, we shall use (32) since this allows an analytical (rather than numerical) approach to be followed.

We shall define our lumped parameter model as

(i) the approximation in (32), which allows a set of coefficients $a_{m}^{*}$ to be determined, and

(ii) having obtained $a_{m}^{*}$, set

$$
a_{m}=a_{m}^{*}
$$

in formulae such as (16) and (17).

The equation for $a_{n}^{*}$,

$$
\alpha^{*} a_{n}^{*}+(2+4 n)\left(\beta^{*}-\alpha^{*}\right) \sum_{m=0}^{\infty} \alpha_{n}^{*} J_{m n}\left(\beta^{*}\right)=\beta^{*} \delta_{n, 0},
$$

follows by substituting (32) into (12), and repeating the steps between (12) and (15). Equation (36) is formally identical to the equation for $a_{n}$ when $z_{1}$ is infinite. In particular, using an obvious notation, $K_{1}^{*}=K_{1}, R_{0}^{*}=R_{0}$, and

$$
h_{j}^{*}=h,+1 /\left[\sum_{l=1}^{N}\left(Z_{l}-Z_{l-1}\right) / K_{k}\right] \text { for } j=0,1 \text {. }
$$


From (33), $a_{n}^{*}$ is a function of only $\alpha^{*}$ and $\beta^{*}$, and so from (17) the important quantity RE is approximated in our lumped parameter model as a function of only $\alpha / \beta, \alpha^{*}$ and $\beta^{*}$. In particular, for large $\beta^{*}$,

whereas for small $\beta^{*}$,

$$
\mathrm{RE} \cong 1-\alpha \beta^{*} /\left\{\beta\left[\alpha^{*}+2\left(\beta^{*}-\alpha^{*}\right) J_{00}\left(\beta^{*}\right)\right]\right\}
$$

$$
\mathrm{RE} \cong 1-\alpha\left[1+8\left(\beta^{*}-\alpha^{*}\right) / 3 \pi\right] / \beta
$$

Our lumped parameter model determines three asymptotic regimes. Firstly, from (38), for small $\beta^{*}$,

$$
\mathrm{RE} \sim 1-h_{0} / h_{1},
$$

which implies that for small $\alpha^{*}$ and $\beta^{*}$ the surface thermal resistances $h_{0}^{-1}$ and $h_{1}^{-1}$ are important. Intuitively, the isotherms are essentially horizontal and continuous within the floor. This follows since in the steady state, temperature differences equal the product of the heat flow and the corresponding thermal resistance. The second regime follows from (37). When $G(0)$ is bounded, and $\beta^{*}$ and $\alpha^{*}$ are large,

$$
\begin{aligned}
\mathrm{RE} & \sim 1-\alpha \beta^{*} / \boldsymbol{\alpha}^{*} \beta \\
& =1-\left[h_{1}^{-1}+\sum_{t=1}^{N}\left(\frac{Z_{t}-Z_{t-1}}{K_{t}}\right)\right] /\left[h_{0}^{-1}+\sum_{i=1}^{N}\left(\frac{Z_{t}-Z_{t-1}}{K_{t}}\right)\right],
\end{aligned}
$$

showing in this case that the total thermal resistance between $T_{N}$ and $T_{0}$ is important. Intuitively a boundary layer develops at the edge of the HFS, with horizontal isotherms away from the vertical boundary layer. A third regime follows when $G(0)$ is infinite, in which case $J_{00}\left(\beta^{*}\right)$ becomes important.

For a finitely thick floor, our lumped parameter model suggests that (40) and (39) provide respectively lower and upper bounds for RE. Alternatively, if $\beta^{*}$ is greater than $\alpha^{*}$,

$$
1 \leqslant a_{0} \leqslant \beta^{*} / \alpha^{*}
$$

a result previously suggested (Weir [6]).

Finally, from (39) and (40), our lumped parameter model predicts that the size of the HFS is important only in the transition region where $\beta^{*}$ is of order unity. Since $a_{0}$ approximately varies between the two bounds in (41) in the transition region, we anticipate that as the ratio $\beta^{*} / \alpha^{*}$ varies away from unity, the behaviour of $a_{0}$ in the transition region becomes more difficult to predict.

\section{Discussion}

This paper has extended the results of a previous paper (Weir [6]). Firstly, we have shown that the function $G(p)$ is fundamental to a mathematical discussion 
of a HFS on a layered medium. Matrix expressions ((21)-(23)), terminating continued partial fractions (27) and a geometric construction, were presented for evaluating $G(p)$, together with a number of algebraic ((24)-(26)) and symmetry properties of $G(p)$.

Secondly, an asymptotic analysis was developed for treating the large $\beta$ case for a homogeneous half-plane of conducting material. The main step was in displacing the original vertical contour in the complex plane a finite number of half integer steps to the left, and observing that this line integral is asymptotic relative to the traversed poles' residues. The leading term in this asymptotic series is in good quantitative agreement for large $\beta$ with our previous numerical results (Weir [6]).

Thirdly, a lumped parameter model was described for multiply-layered media. Undoubtedly this is the least satisfactory, but perhaps the most interesting aspect of this paper.

The lumped parameter model is unsatisfactory because of its non-rigorous nature. It cannot be true generally since it contains only four non-dimensional numbers, whereas arbitrarily many non-dimensional numbers are required to describe a general layered media. However, the lumped parameter model is interesting because in modelling physical systems one is often concerned with particular, rather than the most general cases. Sometimes one seeks a qualitative rather than a quantitative behaviour of a physical system, and if possible, the most important non-dimensional numbers quantifying the system. The lumped parameter model suggests four non-dimensional numbers as being important. Their usefulness is best decided by others. However, a particularly encouraging feature is that for a two-layered finitely thick floor, the lumped parameter model for physically realistic parameter values not only provides the main non-dimensional numbers, but has to date also approximately quantified the relative error $\mathrm{RE}$ in all of over one hundred numerical tests (Harold Trethowen, personal communication). It is hoped to report these results at a later date.

Finally, the variable $p^{*}$ in (33) and (34) has intentionally been left indefined. The approximate agreement between our lumped parameter model and previous empirical approaches referred to above has been obtained by setting $p^{*}$ to unity, but we recognize that more appropriate values of $p^{*}$ can yield improved results. Furthermore, simpler choices for $\alpha^{*}$ and $\beta^{*}$ are also possible. For example,

$$
\alpha^{*}=\alpha+1 / G(0), \quad \beta^{*}=\beta+1 / G(0),
$$

yield similar results to (33) and (34), and avoid evaluation of the tanh functions in $G(p)$. It is hoped to discuss these matters at a later date. 


\section{References}

[1] M. Abramowitz and I. A. Stegun (eds.), Handbook of mathematical functions (Dover, New York, 1972).

[2] C. J. Tranter, Integral transforms in mathematical physics (Methuen, London; John Wiley, New York, 1962).

[3] H. Trethowen and N. Isaacs, "Engineering application of heat flux sensors in buildngs", ASTM Workshop on Building Applications of Heat Flow Sensors, Philadelphia, Sept 22-23, 1983.

[4] E. O. Tuck, "A theory for the design of thin heat flux meters", J. Engrg. Math. 6 (1972), 355-368.

[5] J. R. Wait, Wave propagation theory, (Pergamon Press, New York, 1981).

[6] G. J. Weir, “Insulating circular rugs", J. Austral. Math. Soc. Ser. B 24 (1983), 359-365. 